\title{
Circular RNA profile in liver tissue of EpCAM knockout mice
}

\author{
YANHONG YANG ${ }^{1,2 *}$, SHAOMIN LIU ${ }^{2,3 *}$, ZILI LEI $^{2 *}$, GUIBIN CHEN $^{2 *}$, LI HUANG $^{2,3}$, FEI YANG $^{2,3}$, \\ YUTING LEI ${ }^{2}$, YANYAN LIU ${ }^{2,4}$, LANXIANG YANG ${ }^{2,3}$, WANWAN LIU ${ }^{2}$, LIANGXUE LAI ${ }^{5}$ and JIAO GUO ${ }^{2}$ \\ ${ }^{1}$ The First Affiliated Hospital (School of Clinical Medicine), Guangdong Pharmaceutical University, Guangzhou, \\ Guangdong 510080; ${ }^{2}$ Guangdong Metabolic Disease Research Center of Integrated Chinese and Western Medicine, \\ Institute of Chinese Medicinal Sciences; ${ }^{3}$ School of Traditional Chinese Medicine; ${ }^{4}$ Department of Pathology and \\ Guangdong Key Laboratory for Bioactive Drugs Research, Guangdong Pharmaceutical University, Guangzhou Higher \\ Education Mega Center, Guangzhou, Guangdong 510006; ${ }^{5}$ Key Laboratory of Regenerative Biology, Guangzhou Institutes \\ of Biomedicine and Health, Chinese Academy of Sciences, Guangzhou, Guangdong 510530, P.R. China
}

Received February 28, 2019; Accepted June 26, 2019

DOI: $10.3892 /$ ijmm.2019.4270

\begin{abstract}
Epithelial cell adhesion molecule (EpCAM) is highly expressed during liver development and carcinogenesis, However, its functions and underlying mechanisms remain unclear. Clustered regularly interspaced short palindromic repeats (CRISPRs)/CRISPR-associated protein 9 (Cas9) technology was used in the current study to establish EpCAM $^{-/}$mice. The expression of EpCAM in the livers of the mice at embryonic day (E)18.5 and post-natal day (P)0 was detected by immunofluorescence staining. The expression of genes associated with the development and glycogen metabolism was also assessed by reverse transcription-quantitative PCR. Additionally, the liver tissue of the $\mathrm{EpCAM}^{-/}$and wild-type mice was used for non-coding RNA sequencing. The results of RNA sequencing revealed 11 up-regulated and 12 downregulated circular RNAs (circRNAs). Kyoto Encyclopedia of Genes and Genomes analysis for resource genes determined that the top altered pathways included cell junctions, cell cycle, immune signaling and metabolism. This analysis was also utilized to predict the target association of
\end{abstract}

Correspondence to: Professor Liangxue Lai, Key Laboratory of Regenerative Biology, Guangzhou Institutes of Biomedicine and Health, Chinese Academy of Sciences, 190 Kai Yuan Avenue, Science Park, Guangzhou, Guangdong 510530, P.R. China

E-mail: lai_liangxue@gibh.ac.cn

Professor Jiao Guo, Guangdong Metabolic Disease Research Center of Integrated Chinese and Western Medicine, Institute of Chinese Medicinal Sciences, Guangdong Pharmaceutical University, Guangzhou Higher Education Mega Center, 280 Wai-Huan East Road, Guangzhou, Guangdong 510006, P.R. China

E-mail: gyguoyz@163.com

*Contributed equally

Key words: epithelial cell adhesion molecule, gene knock-out mouse, circular RNA sequencing, liver tissue, development the circRNA-microRNA-mRNA network. The comprehensive liver tissue circRNA expression profiles produced in the present study may help to elucidate the functions and mechanisms of EpCAM during liver development.

\section{Introduction}

Epithelial cell adhesion molecule (EpCAM) is a transmembrane glycoprotein that is involved in several biological processes including cell adhesion, proliferation and differentiation $(1,2)$. It has been demonstrated that $\mathrm{EpCAM}^{-/-}$mice exhibit impaired cell junctions in the intestines, contributing to severe defects and phonotypes similar to human congenital tufting enteropathy (2-4). EpCAM expression is detected in various tissues from early-stage embryos to adults, and in tumors. Huang et al (1) determined the expression pattern, functions and underlying mechanisms of EpCAMs, revealing that the expression pattern of liver EpCAM is particularly complex. EpCAM has been reported to be expressed in hepatoblasts during embryonic development and in adult liver cholangiocytes, but not in hepatocytes $(5,6)$. Previous studies have also identified EpCAM as surface marker of stem cells in normal adult livers (7,8). Furthermore, EpCAM mutant zebrafish exhibit impaired hepatic development due to the activation of the Wnt signaling pathway in the endoderm (9). However, whether EpCAM mutations affect the development of mammalian livers has yet to be elucidated.

The abnormal expression of EpCAM has been associated with different liver diseases. Matsumoto et al (10) reported that proliferating ductal cells (PDCs) expressing EpCAM may lead to the development of hepatocellular carcinoma (HCC) in inflamed livers, indicating the stem/progenitor cell origin of hepatocarcinogenesis. This protein is also highly expressed in premalignant hepatic tissues and EpCAM-positive cells may serve as HCC cancer stem cells (11-13). Mani et al (14) demonstrated that EpCAM underwent regulated intramembrane proteolysis in hepatitis B virus (HBV)-replicating cells. It was also revealed that the activation of Wnt signaling may lead to HBV-associated HCC. It has been reported that bile acids induce the expression of long non-coding RNA H19, and 
the activation of hepatic H19RNA may promote cholestatic liver fibrosis in mice via the ZEB1/EpCAM signaling pathway in mice (15). Although the expression pattern of EpCAM has been studied, the functions and mechanisms of EpCAM in liver development and disease remain unclear.

Circular RNAs (circRNAs), a novel type of non-coding RNAs (ncRNAs) with a covalently closed-loop structure generated by back splicing, play important roles in several biological processes, including proliferation, apoptosis, development and aging $(16,17)$. circRNAs may also serve as potential clinical biomarkers for various diseases, including rheumatoid arthritis, breast cancer, cardiovascular diseases and osteoarthritis (18-21). It has been demonstrated that a number of circRNAs are differentially expressed between HCC and normal liver tissues, which may be closely associated with the development and prognosis of HCC (22). The altered expression of circRNAs has also been implicated in hepatic steatosis and non-alcoholic fatty liver disease $(23,24)$.

The aforementioned studies indicate that the expression of circRNAs may be involved in the functions of EpCAM and that circRNAs may serve as biomarkers during liver development and associated diseases. In the current study, the effects of EpCAM on the expression profiles of circRNAs in the livers of $\mathrm{EpCAM}^{-/-}$mice were investigated by high-throughput sequencing. In addition, circRNA-microRNA (miRNA or miR)-mRNA networks were assessed by target analysis. The present study identified several novel circRNAs in the livers of $\mathrm{EpCAM}^{-/}$mice. These results may therefore enhance our understanding of the functions of EpCAM during liver development and in associated diseases.

\section{Materials and methods}

Generation of EpCAM ${ }^{-/}$mice. All animal experiments were approved by the Committee on the Laboratory Animal Care and Use of Guangdong Pharmaceutical University. Stable genetic $\mathrm{EpCAM}^{-/}$mice were obtained using the clusters of regularly interspaced short palindromic repeats (CRISPRs)/CRISPR-associated protein 9 (Cas9) gene editing technology. The method was as follows: The GGGCGATCCAGAACAACGAT sequence in exon 2 of EpCAM was identified as guide RNA (gRNA) by Vector NTI11 software (version 11.5.1, Invitrogen) (Fig. 1A). The gRNA and Cas9 gene constructed in vitro were transfected into the fertilized ova of C57BL/6 mice via electrotransfection, which was subsequently transplanted into the oviducts of surrogate 10-week old ICR ( $\mathrm{n}=12$; body weight, 30-34 g) female mice. Fertilized ova were obtained by mating 10-15-week old C57BL/6 males ( $\mathrm{n}=10$; body weight, 20-30 g) with 6-week-old C57BL/6 females ( $\mathrm{n}=29$; body weight, 18-20 g). F0 mice with successful gene knockout, confirmed by gene sequencing, were mated with wild-type (WT) 8-12-week-old C57BL/6 mice $(n=32$; body weight, 20-29 g). F1 hybrid mice were then screened out. Female and male mice with identical knockout sequences were selected for breeding F2 mice. Heterozygous F2 mice with stable genetic characteristics were then selected for breeding and conservation. Homozygous mice with the knockout gene were selected for further analysis. All the mice were kept in an SPF mouse facility, at $22^{\circ} \mathrm{C}, 60-65 \%$ humidity, with free access to food and water. All mice were from Hunan SJA Laboratory Animal Co. Ltd.
Animal tissue collection for circRNA sequencing. WT and EpCAM $^{-/-}$mice were sacrificed on the first day after birth, after which murine livers were weighed, frozen in liquid nitrogen, and stored at $-80^{\circ} \mathrm{C}$ for preservation. The livers of every 3 mice (at least $200 \mathrm{mg}$ ) were collected as 1 sample. The control group (WT) and the EpCAM $^{-/}$group had 3 samples each.

Hematoxylin and eosin $(H \& E)$ and immunofluorescence staining. The mouse tissues were fixed in $4 \%$ paraformaldehyde at $4^{\circ} \mathrm{C}$ overnight for $\mathrm{H} \& \mathrm{E}$ (Leagene Biotechnology) and immunofluorescence staining H\&E staining was performed on $4-\mu \mathrm{m}$-thick sections, stained with hematoxylin for $3 \mathrm{~min}$ and eosin for $20 \mathrm{sec}$ at room temperature. Immunofluorescence staining was performed on frozen sections. Tissues were embedded in optimal cutting temperature compound (OCT) (Sakura Finetek) after fixing, and $7-\mu \mathrm{m}$-thick sections were mounted on the slides using the frozen section machine (LEICA CM1860). The sections were boiled in $10 \mathrm{mM}$ citric acid (Merck) at $\mathrm{pH} 6.0$ for $5 \mathrm{~min}$, and then were exposed to $1 \%$ bovine serum albumin (BSA; Sigma) in phosphate-buffered saline (PBS; HyClone) containing $0.1 \%$ Tween-20 to block non-specific sites. Anti-rabbit EpCAM antibody (cat. no. ab71916; Abcam) was incubated with the sections at $4^{\circ} \mathrm{C}$ overnight at a dilution of 1:200, and AlexFluor 488 secondary antibody (cat. no. A21206; Thermo fisher Scientific, Inc.) was incubated with the sections at room temperature for $1 \mathrm{~h}$ at a dilution of 1:1,000. Images of H\&E staining were captured using the PerkinElmer Automated Quantitative Pathology System, and the images of immunofluorescence staining were analyzed using an Olympus confocal microscope (FV3000).

RNA extraction and library construction. circRNA isolation, library construction, circRNA sequencing and bioinformatics analysis were performed by the Gene Denovo Biotechnology Co. Total RNA was extracted using TRIzol reagent (Life Technologies) and, ribosomal RNA (rRNA) [Ribo-Zero Gold (Human/Mouse/Rat) kit, Illumina] was removed to retain mRNA and ncRNA. Enriched mRNAs and ncRNAs were separated into short fragments and reverse transcribed into cDNA (NEB\#7490 kit, New England Biolabs) with random primers. Second-strand cDNA was then synthesized (NEB\#7490 kit, New England Biolabs) using DNA polymerase I, RNaseH and dNTPs (NEB\#7490 kit, New England Biolabs). The cDNA fragments were purified using the QiaQuick PCR extraction kit (Qiagen), end repaired, poly(A) added, and ligated into Illumina sequencing adapters (NEB\#7490 kit, New England Biolabs). Uracil-N-Glycosylase (NEB\#7490 kit, New England Biolabs) was then used to digest the second-strand cDNA. Digested products were size selected via agarose gel electrophoresis (Sigma), PCR-amplified, and sequenced using Illumina HiSeqTM 4000 (Illumina).

circRNA sequencing analysis. Reads were further filtered to remove those of low quality reads containing adapters or reads with $>10 \%$ of unknown nucleotides. Reads were then mapped to rRNA of mice (Genome version GRCm38.p5, http://asia. ensembl.org/info/about/species.html) and the reference genome was established using Bowtie2 (version 2.2.8, http://bowtie-bio. sourceforge.net/bowtie2/index.shtml) and TopHat2 

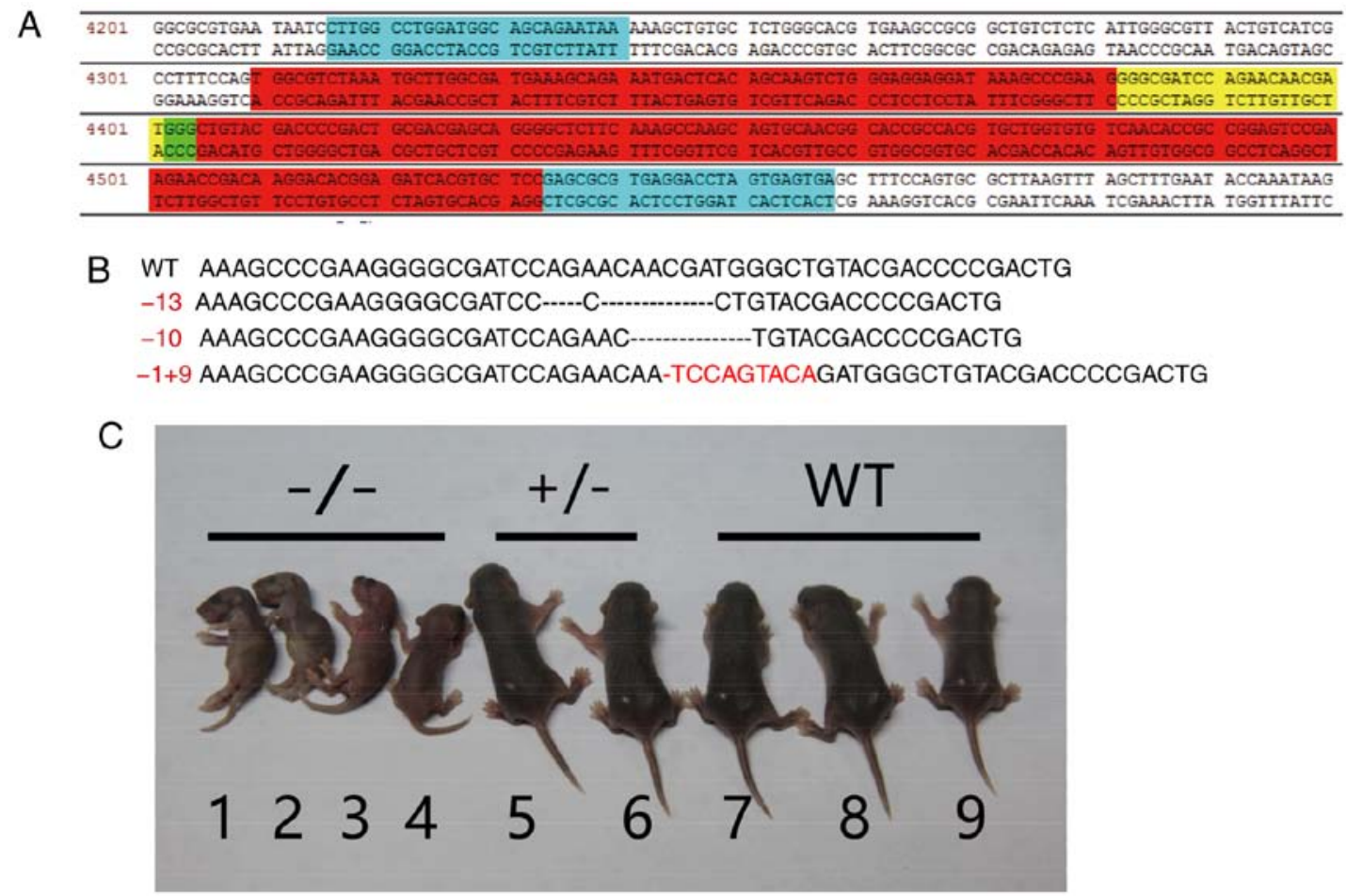

Figure 1. Generation of EpCAM knockout mice. (A) The GGGCGATCCAGAACAACGAT sequence in exon 2 (yellow) of EpCAM was identified as guide RNA using Vector NTI11 software. Red, blue and green represent the exon, PCR primers and the protospacer adjacent motif site, respectively. (B) Three types of EpCAM gene knockout mice with termination codon mutations were obtained, and named as $-13,-10$ and $-1+9$ bp mutants. (C) EpCAM ${ }^{--}$mice generated by clusters of regularly interspaced short palindromic repeats/CRISPR associated protein 9 gene editing technology were significantly smaller than heterozygous and wild-type mice at day 4 after birth. EpCAM, epithelial cell adhesion molecule.

(version 2.1.1, http://ccb.jhu.edu/software/tophat/index.shtml) software $(25,26)$, respectively. Mapped reads were abandoned and unmapped reads were collected for circRNA identification. Each end of the unmapped reads $(20 \mathrm{bp})$ was extracted and aligned to the reference genome to find unique anchor positions within splice site. Anchor reads that aligned in the reversed orientation (head-to tail) were then analyzed via Find_circ software (version 1) (27) to identify circRNAs. The type, chromosome distribution and length distribution of the identified circRNAs were then further analyzed. The functions of circRNA source genes were also analyzed via functional enrichment analysis as shown below.

Analysis of differentially expressed circRNAs and database annotation. Differentially expressed circRNAs were identified using the edgeR package (http://www.r-project.org/). circRNAs were then blasted against the circBase for annotation and those that could not be annotated were defined as novel circRNAs. A fold change $\geq 2$ and P-value $<0.05$ in the comparison between different groups were considered to indicate statistically significant differences.

Gene ontology (GO) and kyoto encyclopedia of genes and genomes (KEGG) pathway analysis. GO is a gene functional classification system that defines the properties and functions of genes in different organisms. GO has 3 ontologies: Molecular function, cellular component and biological process. All the source genes were mapped to GO terms in the GO database (http://www.geneontology.org/) and gene numbers were calculated for every term, with FDR $\leq 0.05$ as a threshold.
KEGG pathway analysis (https://www.kegg.jp/) identifies significantly enriched pathways in source genes compared with the whole genome, which helps to further understand the functions of genes. The calculating formula is the same as GO analysis, and pathways with FDR $\leq 0.05$ were defined as significantly enriched.

Integrated analysis of circRNAs-miRNAs-mRNAs. StarBase (v2.0) software was used to predict the target miRNAs of known circRNAs. Mireap (https://sourceforge.net/projects/mireap/), Miranda (v3.3a, http://miranda.org.uk/) and TargetScan (v7.0, http://www.targetscan.org) were also used to predict the target miRNAs of novel circRNAs. The circRNA-miRNA-mRNA pathway regulatory network was analyzed via miRTarBase (v6.1, http://mirtarbase.mbc.nctu.edu.tw/php/index.php), and the resulting correlation was visualized by Cytoscape (https://cytoscape.org/).

Reverse transcription-quantitative PCR (RT-qPCR). Total RNA was extracted from the liver samples using TRIzol reagent (Invitrogen, Thermo Fisher Scientific, Inc.), and subjected to reverse transcription using the PrimeScript ${ }^{\mathrm{TM}} \mathrm{RT}$ Reagent kit (Takara). All primers of development and metabolism associated genes are listed in Table SI [produced by Sangon Biotech (Shanghai) Co., Ltd.]. qPCR was performed using the SYBR Premix Ex Taq kit (Takara) with the PikoReal PCR system (Thermo Fisher Scientific, Inc.,) under the following conditions: $95^{\circ} \mathrm{C}$ for $30 \mathrm{sec}$, followed by 40 cycles at $95^{\circ} \mathrm{C}$ for $5 \mathrm{sec}$, $60^{\circ} \mathrm{C}$ for $20 \mathrm{sec}$ and $65^{\circ} \mathrm{C}$ for $15 \mathrm{sec}$. GAPDH was used as a reference gene. 
To confirm the expression of circRNAs, qPCR was performed under the following sequential conditions: $95^{\circ} \mathrm{C}$ for $5 \mathrm{~min}, 40$ cycles at $95^{\circ} \mathrm{C}$ for $10 \mathrm{sec}$ and $60^{\circ} \mathrm{C}$ for $34 \mathrm{sec}$. All the primers of circRNAs were listed in Table SII (produced by Geneseed Biotechnology Co., Ltd.). $\beta$-actin was used as the reference gene. PCR products were tested via electrophoresis on $2 \%$ agarose gels and bands were extracted and used for Sanger sequencing.

Statistical analysis. Statistical differences were determined using SPSS 23.0 software. An independent-sample t-test was used and the data were presented as the means \pm SD. $\mathrm{P}<0.05$ was considered to indicate a statistically significant difference.

\section{Results}

Generation of EpCAM knockout mice. Three types of EpCAM gene knockout mice with termination codon mutations were obtained and named $-13 \mathrm{bp},-10 \mathrm{bp},-1+9 \mathrm{bp}$ mutant, respectively (Fig. 1B). The phenotypes of these mutants were identical. The $-1+9$ bp mutant mice were selected for further analysis. The phenotype of the EpCAM knockout mice obtained via CRISPR/Cas9 was identical to that obtained by the traditional gene targeting technique of our previous study (2). As presented in Fig. 1C, the EpCAM ${ }^{-/-}$mice generated via CRISPR/Cas9 gene editing were smaller than the heterozygous and WT mice at day 4 after birth (Fig. 1C). The 89.36\% (42/47) of the mutant mice died within 1 week after birth, and all the mutant mice died within 12 days. This result was similar to previous studies, and the mice died of intestinal erosion as EpCAM knockout affects the intestinal tight junction (2-4).

Expression pattern of glycogen-related genes in the livers of EpCAM KO mice. As shown in Fig. 2A, the expression level of EpCAM was significantly decreased in the EpCAM knockout mice. The results of immunofluorescence staining also demonstrated that EpCAM was primarily expressed in the liver cholangiocytes of WT mice at E18.5 (Figs. 2B and S1A) and at P0 (Fig. S1C). H\&E staining revealed no marked differences in the livers of the $\mathrm{EpCAM}^{-/}$and WT mice at E18.5, although some $\mathrm{EpCAM}^{--}$mice exhibited reduced cytoplasmic vacuolation when compared with WT mice (Fig. 2C), indicating defects in hepatic glycogen storage (28). In addition, no marked differences were observed following H\&E staining in the livers of EpCAM- ${ }^{--}$and WT mice at P0. The EpCAM ${ }^{-1-}$ murine livers at $\mathrm{P} 4$ exhibited marked vacuolation compared with those of the WT mice Fig. S1D.

The expression levels of development associated genes, including marker of hepatoblast delta-like 1 homologue (DLK1; Fig. 2D), hepatocyte albumin (ALB), CD26 and H19 (Fig. 2E), hepatic oval cell CD34 and thymus cell antigen 1 (Thyl) (Fig. 2F), Wnt signal pathway axis inhibition protein 2 (Axin2) and leucine rich repeat containing G-protein coupled receptor 5 (Lgr5) (Fig. 2G) and cholangiocyte hepatocyte nuclear factor $1 \beta$ (Hnf1 $\beta$ ), cytokeratin 19, CD133 and SRY box 9 (Sox9; Fig. 2H) were further assessed by RT-qPCR. The results revealed that while the expression of CD34 was increased in the knockout mice, the level of Axin2 was significantly decreased. The expression of Hnf $1 \beta$ was also increased, but not significantly $(\mathrm{P}=0.09)$.
Since some $\mathrm{EpCAM}^{-/-}$mice demonstrated reduced cytoplasmic vacuolation of the liver, suggesting defects in hepatic glycogen storage (28), the expression of glycogen-associated genes was also determined (Fig. 3). Glycogen phosphorylase and glucose-6-phosphatase (G6PC) were determined to be associated with glycogenolysis in the liver $(29,30)$, and the expression of G6PC was decreased in the knockout mice ( $\mathrm{P}=0.08$, Fig. $3 \mathrm{~A})$. The expression levels of two glycogen synthesis-associated genes, UDP-glucose pyrophosphorylase 2 (Ugp2) and glucokinase (GCK) were significantly decreased $(\mathrm{P}=0.08$, Fig. 3B). The expression levels of various genes that regulate glycogen catabolism, including protein phosphatase 1 regulatory subunit $3 \mathrm{~B}$ (PPP1R3B), protein phosphatase 1 catalytic subunit $\alpha$ (PPC1CA), common $\beta$ subunit of phosphorylase kinase (PHKB), phosphorylase kinase catalytic subunit, gamma 2 (PHKG2) remained unaltered (Fig. 3C). Additionally, no changes in the expression of PAS domain containing serine/threonine kinase (PASK), which regulates glycogen synthesis in the liver (31), were observed (Fig. 3D). The expression of solute carrier family 2 (facilitated glucose transporter), member 2 [SLC2A2; also known as glucose transporter-2 (GLUT-2)], a gene associated with disorders of glycogen storage (32), was significantly increased (Fig. 3E).

Overview of circRNAs in the livers of EpCAM-/- mice. According to the sequence data, 526,000,032 clean reads $\left(255,388,976\right.$ for $\mathrm{EpCAM}^{-/-}$mice and 270,611,056 for WT mice) were generated. Following the removal of the low quality, adapter-containing and poly-N containing reads, 518,617,322 high quality clean reads $\left(251,809,406\right.$ from EpCAM $^{-/}$mice and 266,807,916 from WT mice) were obtained (Table SIII). A total of 3,984 circRNAs were identified, including 292 known circRNAs and 3,692 novel circRNAs. The majority of the circRNAs were 300-400 nucleotides in length (Fig. 4A). The length distribution of known circRNAs and novel circRNAs are presented in Fig. S2A and B, respectively. Different types of circRNAs were identified, including annot_exons, antisense, exon_intron, intergenic, intronic and one_exon circRNAs. Among these, the most common type was annot_exon followed by one_exon (Fig.4B-C). The different types of known and novel circRNAs are presented in Fig. S2C and D, respectively, of which, the first and second most common types were also annot_exon and one_exon. In addition, the chromosome distributions of known circRNAs and novel circRNAs are presented in Fig. S2E and F, respectively.

To elucidate the functions of circRNAs, all source genes were mapped to terms in the GO database and compared with the background. GO has 3 ontologies including biological process, cellular component and molecular function. In the biological process group, the majority of genes were associated with cellular process, metabolic process and single-organism process. In the cellular component group, the majority of genes were associated with cell, cell part and organelle. Additionally, in the molecular function group, the majority of genes were associated with binding and catalytic activity (Fig. 4D).

KEGG assignments were used to classify the functional annotations of source genes to further understand the biological functions of circRNAs. The top 20 pathways are presented in Fig. 4E and included ubiquitin mediated proteolysis, thyroid hormone signaling, adherents junction, cell cycle and cell senescence, regulation of actin cytoskeleton, and insulin signaling pathway. 


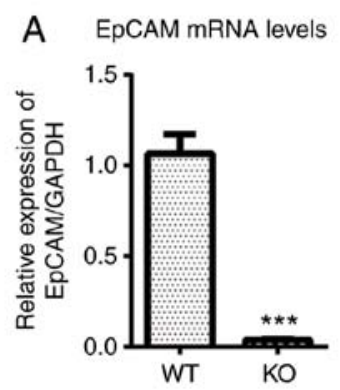

C

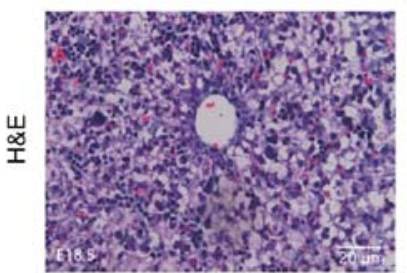

B

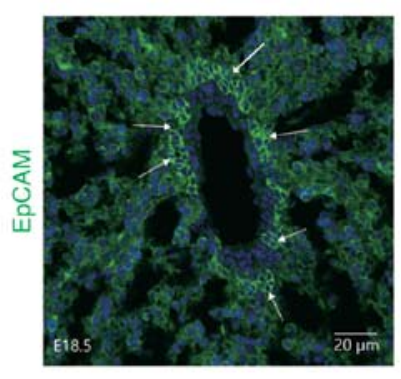

$\mathrm{KO}$

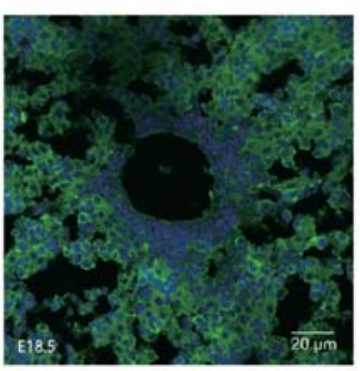

KO

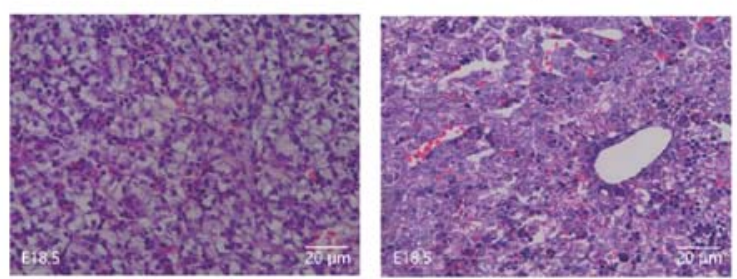

D

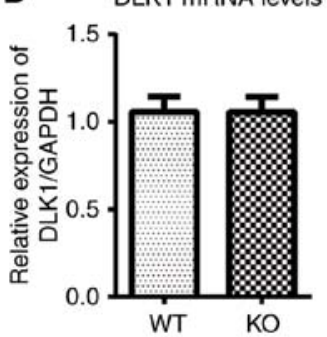

F
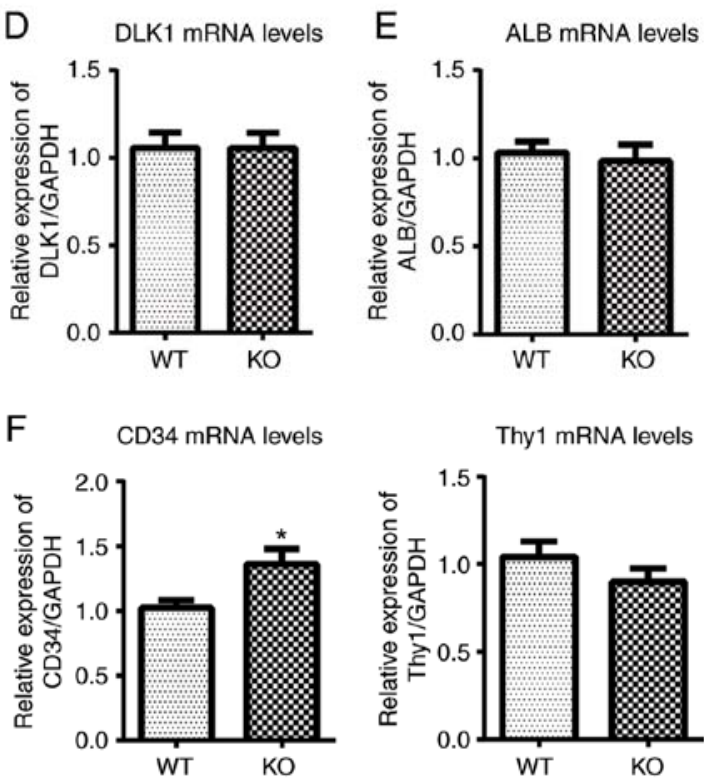

$\mathrm{H}$

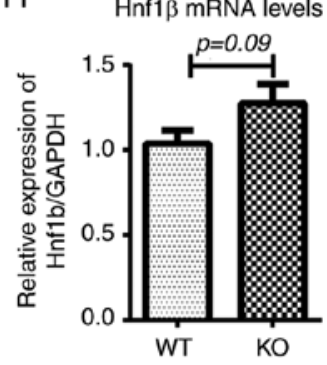

Thy1 mRNA levels

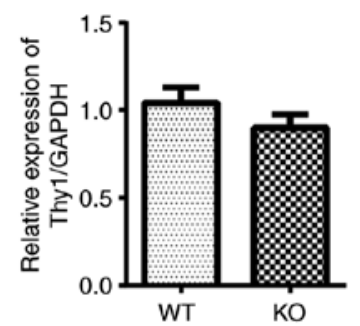

CK19 mRNA levels

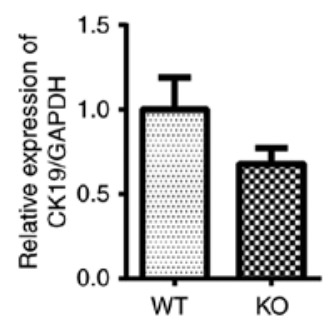

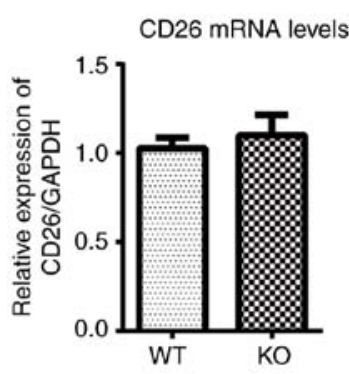

G

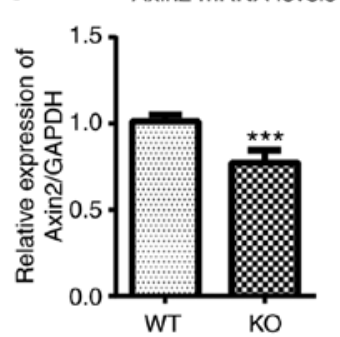

CD133 mRNA levels

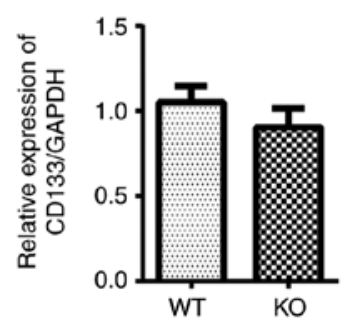

H19 mRNA levels

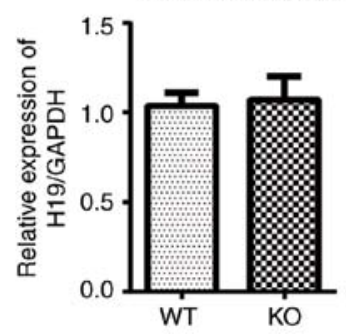

Lgr5 mRNA levels

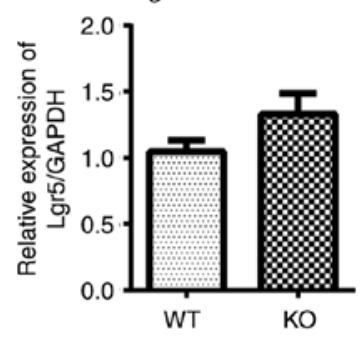

Sox9 mRNA levels

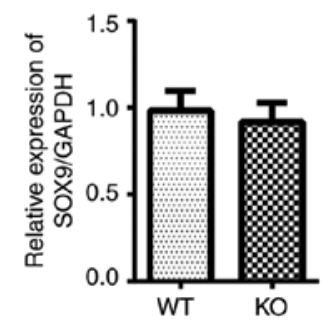

Figure 2. H\&E staining and immunofluorescence staining of EpCAM, and the expression of development-associated genes at the mRNA level in the livers of $\mathrm{EpCAM}^{-/-}$mice at embryonic day (E)18.5, as detected by reverse transcription-quantitative PCR. (A) The expression of EpCAM was significantly decreased in the livers of EpCAM ${ }^{-/}$mice at E18.5. (B) Immunofluorescence staining of EpCAM in the livers of EpCAM ${ }^{-/-}$mice and WT mice at E18.5. (C) H\&E staining of $\mathrm{EpCAM}^{-/}$murine livers and WT mice of E18.5. The expression levels of (D) DLK1, (E) ALB, CD26 and H19, (F) CD34 and Thy1, (G) Axin2 and Lgr5, (H) Hnf1 1 , CK19, CD133 and Sox9 were determined. ${ }^{*} \mathrm{P}<0.05$ and ${ }^{* * *} \mathrm{P}<0.001$, vs. WT mice. H\&E, hematoxylin and eosin; EpCAM, epithelial cell adhesion molecule; WT, wild-type; DLK1, marker of hepatoblast delta-like 1 homologue; ALB, albumin; Axin2, axis inhibition protein 2; Lgr5, leucine rich repeat containing G-protein coupled receptor; Hnf1 $\beta$, hepatocyte nuclear factor 1 $\beta$; CK19, cytokeratin 19; Sox9, SRY box 9.

circRNA expression profiles in the livers of EpCAM ${ }^{-1}$ mice. The differences in circRNA expression between the livers of WT and $\mathrm{EpCAM}^{-/-}$mice were compared and those with a $\log _{2}$ fold change $>1$ and P-value $<0.05$ were considered statistically significant. A total of 11 upregulated circRNAs and 12 downregulated circRNAs were identified in the livers 
A PYGL mRNA levels

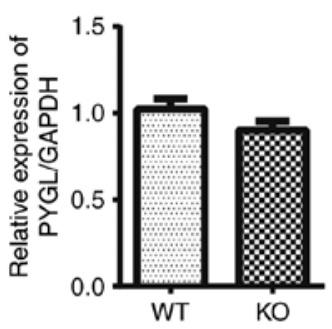

C PPP1R3B mRNA levels

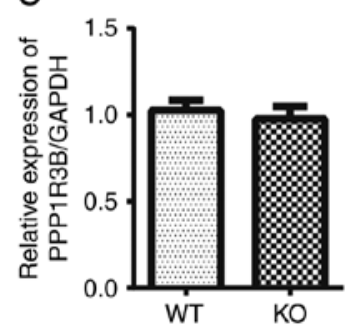

G6PC mRNA levels

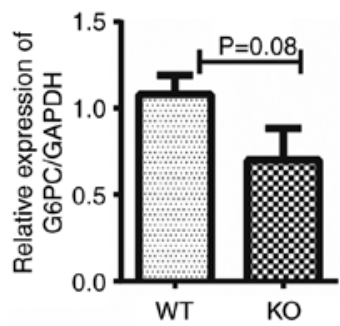

PPP1CA mRNA levels

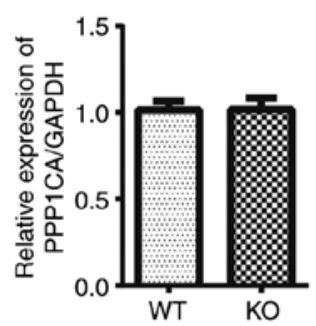

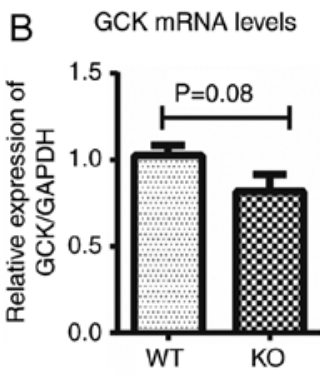

PHKB mRNA levels

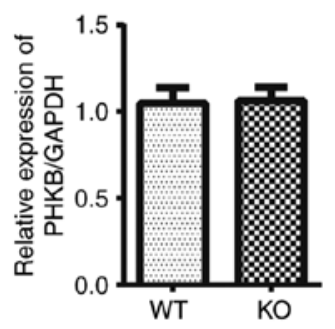

UGP2 mRNA levels

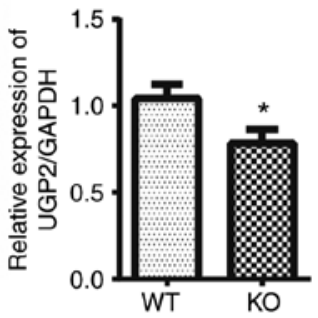

PHKG2 mRNA levels

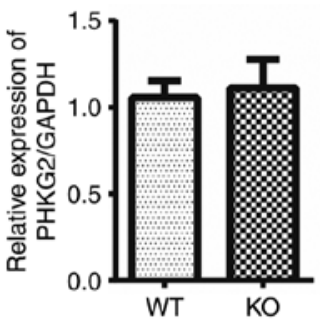

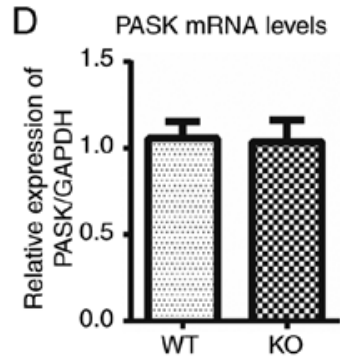

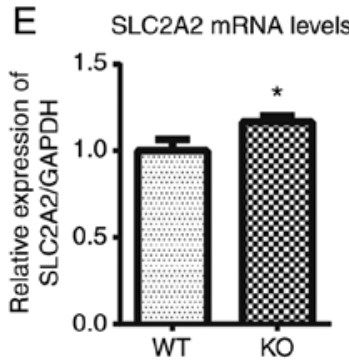

Figure 3. Expression of glycogen-associated genes at the mRNA level in the livers of EpCAM ${ }^{-/}$mice at E18.5, as detected by reverse transcription-quantitative PCR. The expression levels of (A) PYGL and G6PC, (B) GCK and UGP2, (C) PPP1R3B, PPP1CA, PHKB and PHKG2, (D) PASK and (E) SLC2A2 were determined. "P<0.05, vs. WT mice. EpCAM, epithelial cell adhesion molecule; WT, wild-type; PYGL, glycogen phosphorylase; G6PC, glucose-6-phosphatase; GCK, glucokinase; UPG2, UDP-glucose pyrophosphorylase 2; PPP1R3B, regulatory subunit of phosphoprotein phosphatase-1, PPC1CA, protein phosphatase 1 catalytic subunit $\alpha$; PHKB, common $\beta$ subunit of phosphorylase kinase; PHKG2, $\gamma$ subunit hepatic phosphorylase kinase; PASK, PAS domain containing serine/threonine kinase; SLC2A2, solute carrier family 2 member 2.

of EpCAM ${ }^{-/-}$mice compared with the WT group (Fig. 5A), including 6 known circRNAs and 17 novel circRNAs. The basic information of the upregulated and downregulated circRNAs and their predicted target miRNAs is presented in Tables I and II, respectively.

The results of hierarchical clustering, which is one of the most commonly used clustering techniques for gene expression analysis, revealed the distinguishable circRNA expression profiles between the WT and EpCAM ${ }^{-/}$mice (Fig. 5B).

The results of KEGG pathway analysis demonstrated that the top 20 pathways were the mRNA surveillance pathway, fat digestion and absorption, insulin signaling, Hippo signaling and cell senescence, as well as others (Fig. 5C).

The results of GO analysis determined that in the biological process group, the majority of source genes of differentially expressed circRNAs were associated with cellular process, single-organism process, metabolic process and developmental process. In the cellular component group, the majority of genes were associated with cell, cell part, organelle organelle part and membrane-enclosed lumen. Furthermore, in the molecular function group, the majority of genes were associated with binding and catalytic activity (Fig. 5D).

Validation of circRNA expression profiles in the livers of $E p C A M^{--}$mice. To validate circRNA profiles, 3 circRNAs were randomly selected and amplified by RT-qPCR. The results revealed that the expression levels of novel_circ_00176 and _circ_002561 were significantly increased in the livers of $\mathrm{EpCAM}^{-/-}$mice compared with those of WT mice. Furthermore, the expression of novel_circ_00189 was decreased in the livers of EpCAM ${ }^{-/}$mice (Fig. 6A). These results were in agreement with the RNA-seq data. The PCR products were subsequently analyzed using agarose gels to validate single DNA amplifications (Fig. 6B). In addition, the identity of 3 selected circRNAs were further confirmed by Sanger sequencing (Fig. 6C).

Prediction of target association of circRNA-miRNA-mRNA network. To confirm the functions of circRNAs as 'miRNAs sponges', the mmu-miR-302b-5p and mmu-miR-691 binding sites of novel_circ_000189; the mmu-miR-200b-3p and mmu-miR-195a-5p binding sites of novel_circ_000176; and the mmu-miR-200a-3p and mmu-miR-466b-5p binding sites of novel_circ_002561 are presented in Fig. 7. All the established miRNA binding sites of the 3 selected circRNAs are presented in Figs. S3-S5. The regulatory circRNA-miRNA-mRNA networks of the 3 circRNAs were further established. In novel_circ_002561, the target genes associated with the Wnt signaling pathway [(Wnt family member 3a (Wnt3a), Lgr5, transcription factor (Tcf) 3 and Tcf 4 and others], cell junctions (cadherin 1, catenin beta-1 and cell adhesion molecule 1 and 

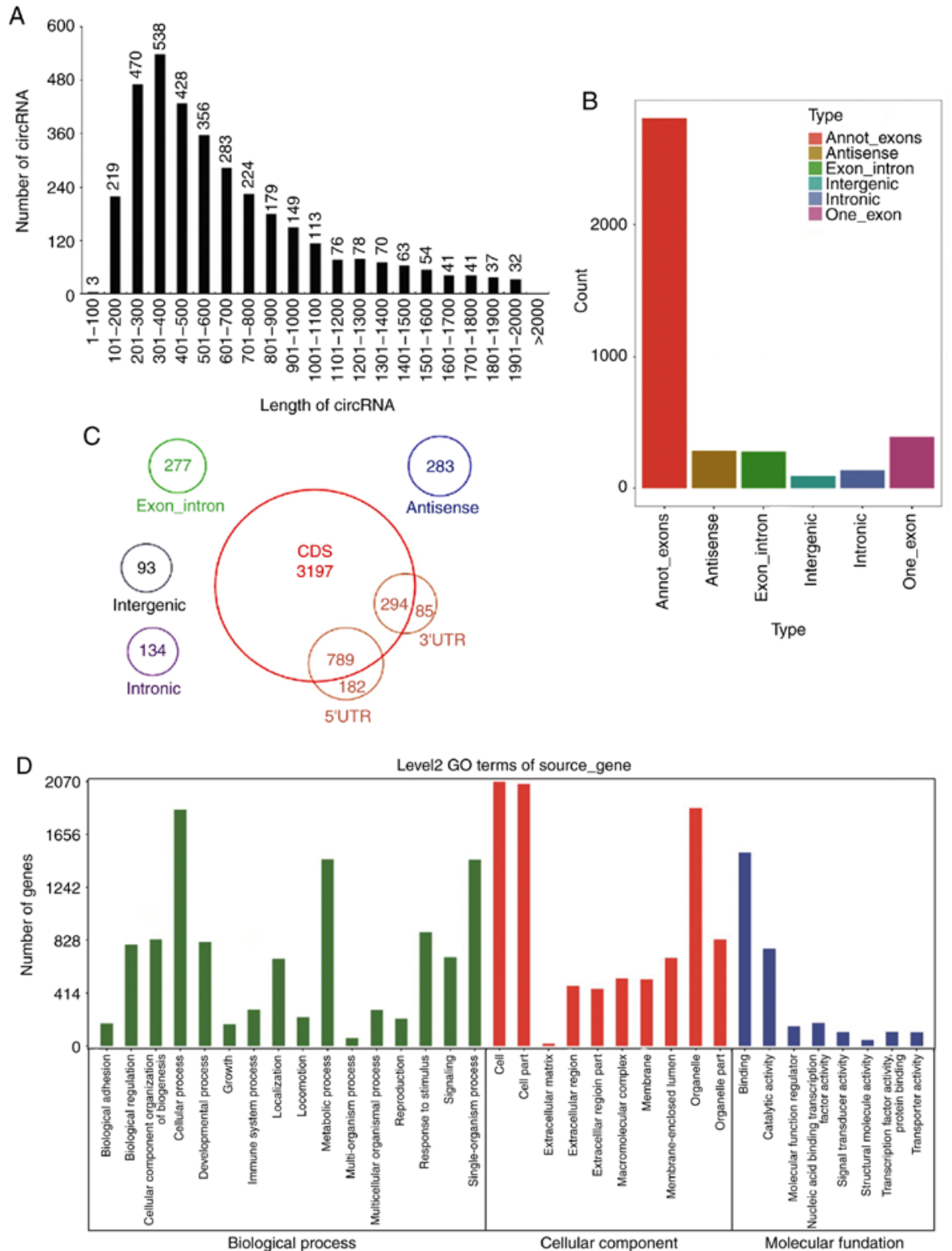

E

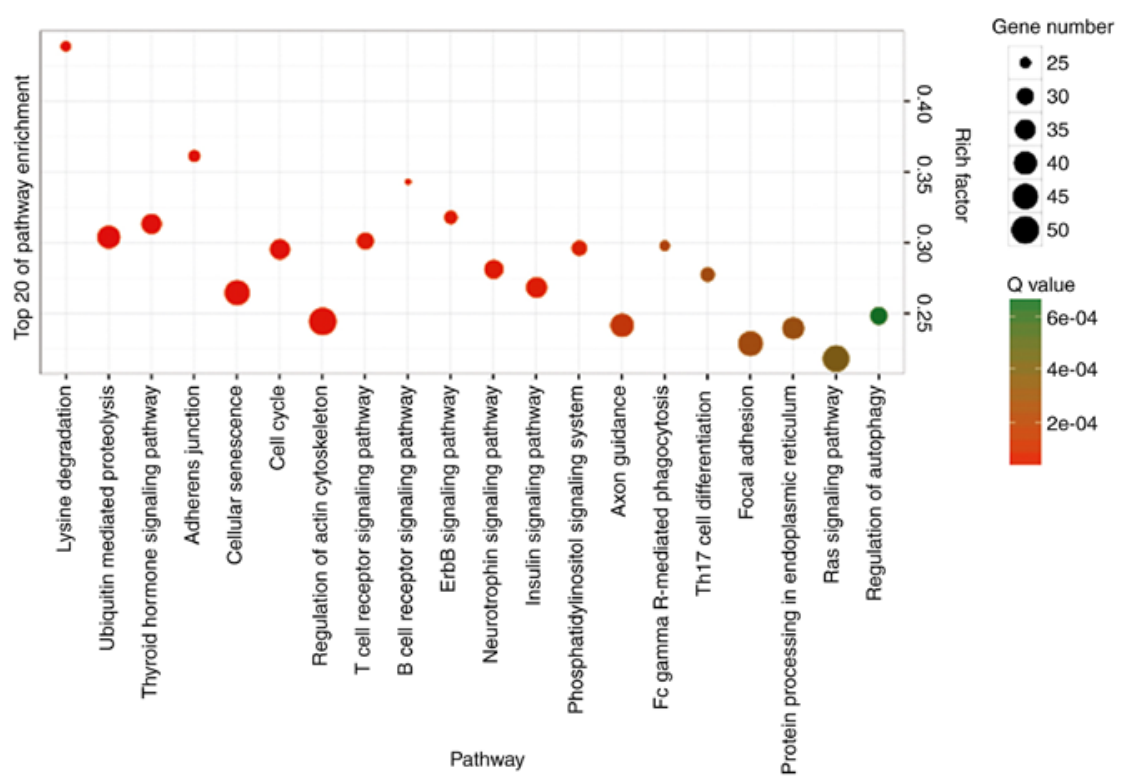

Figure 4. Overview of circRNAs in the livers of EpCAM ${ }^{-/}$mice. (A) The length distribution of circRNAs. (B and C) the types of circRNAs identified. (D) The presentation of GO classification of the source genes of the circRNAs and (E) Kyoto Encyclopedia of Genes and Genomes assignments of circRNA source genes. circRNA, circular RNA; EpCAM, epithelial cell adhesion molecule. 

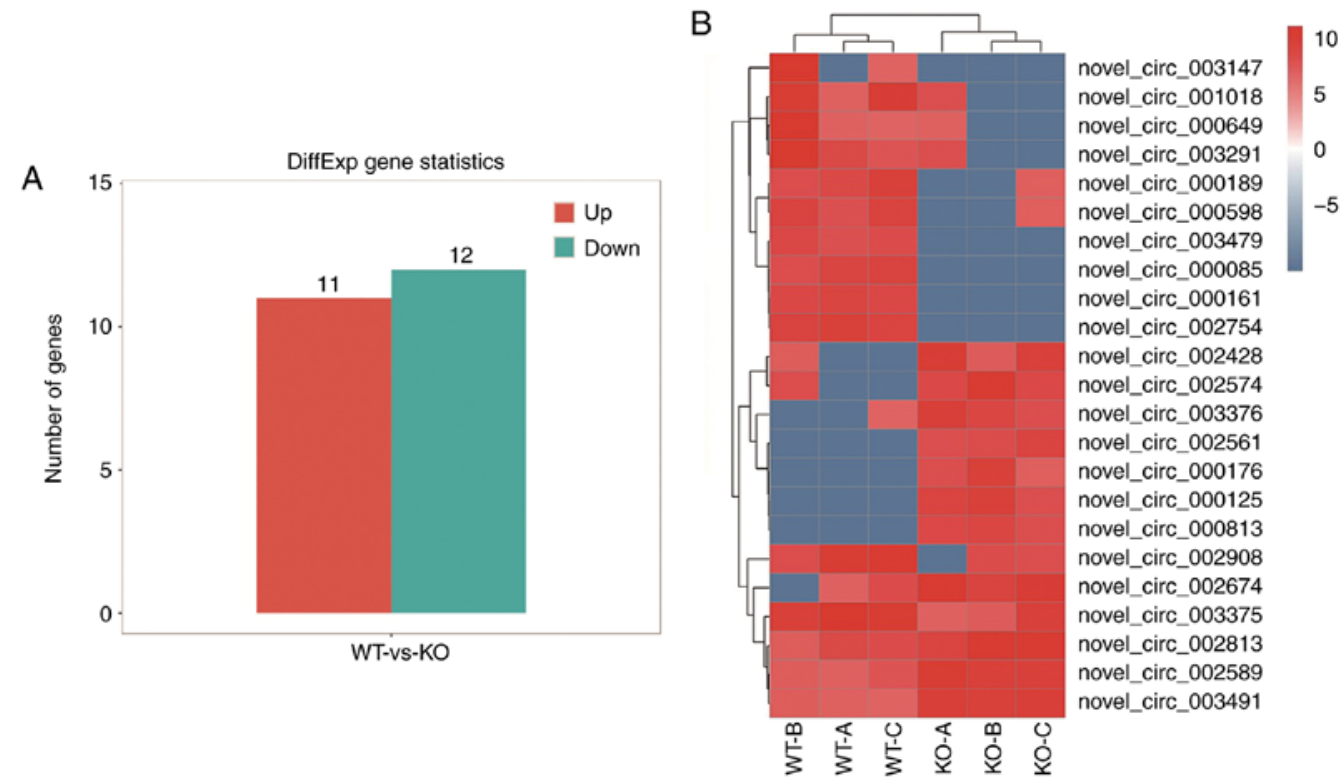

C

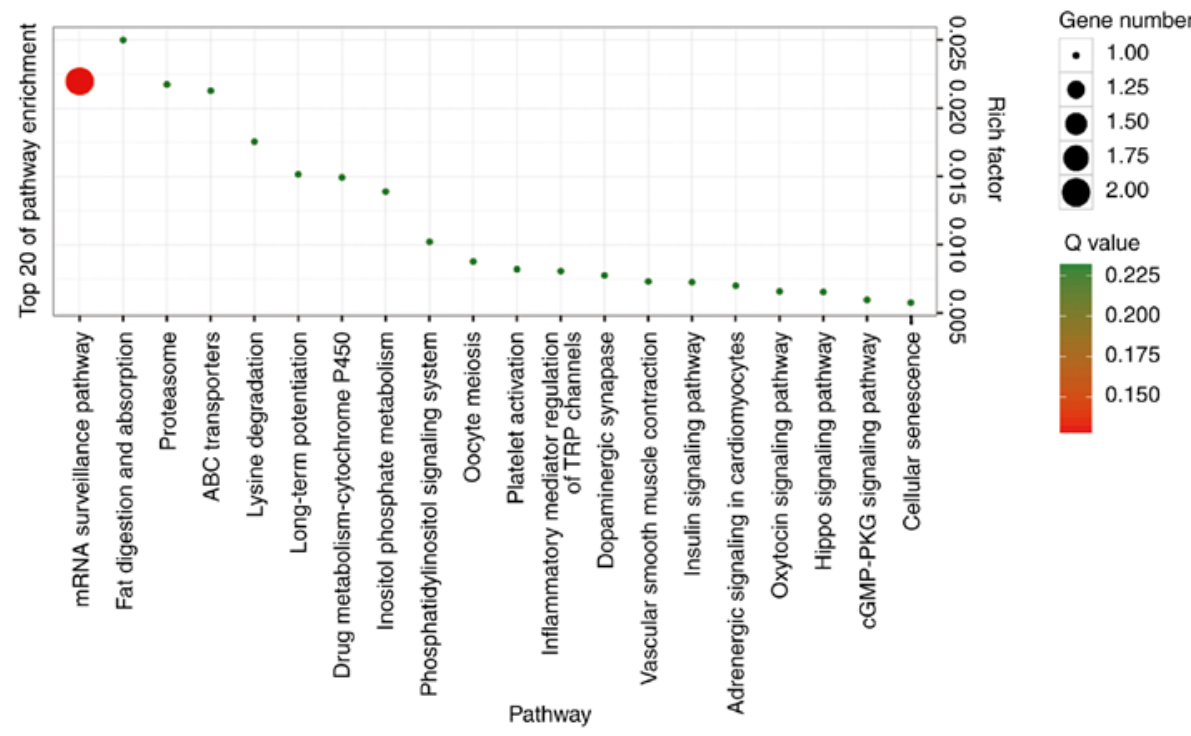

D

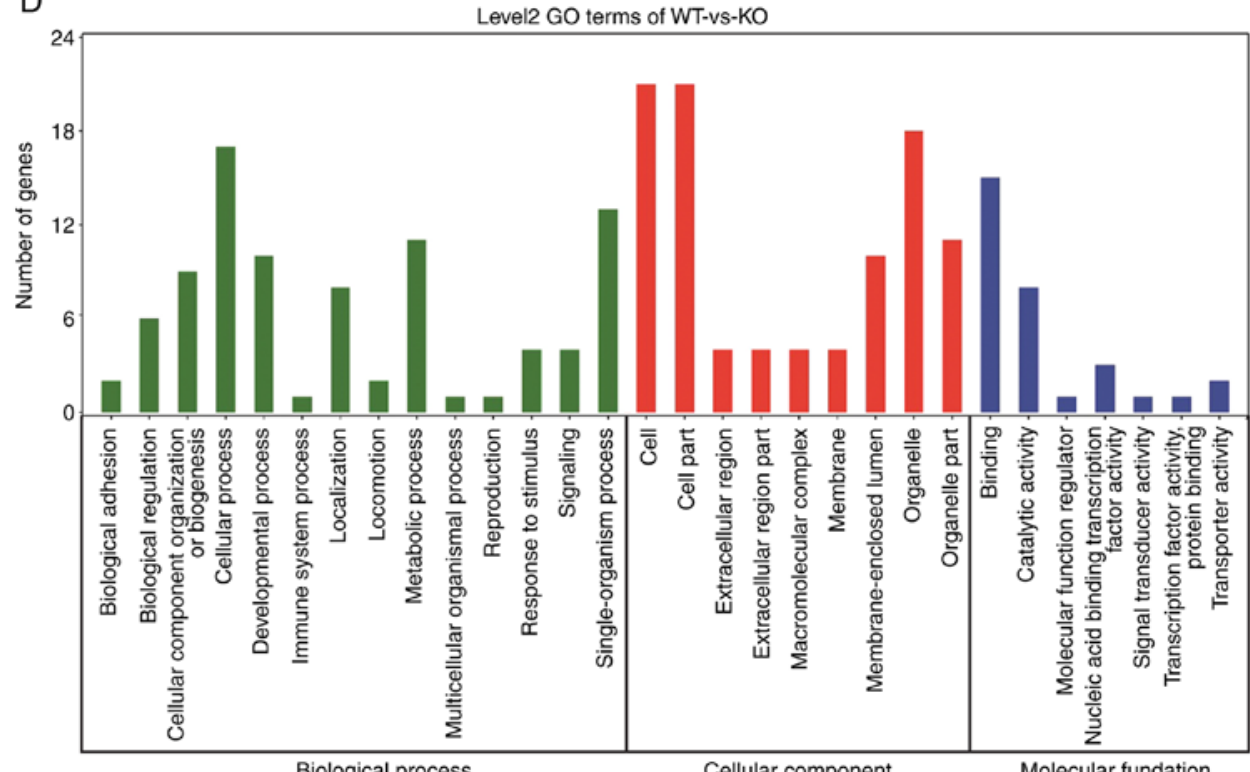

Figure 5. circRNA expression profiles in the livers of EpCAM ${ }^{-1}$ mice. (A) The circRNA expression profile of EpCAM ${ }^{-/}$murine livers. (B) Heatmap of the distinguishable circRNA expression profiles between the wild-type and the EpCAM ${ }^{-/}$groups. (C and D) Kyoto Encyclopedia of Genes and Genomes assignments and GO classification of the source genes of the differentially expressed circRNAs. circRNA, circular RNA; EpCAM, epithelial cell adhesion molecule. 
Table I. Basic information and predicted targets of up-regulated circRNAs.

\begin{tabular}{|c|c|c|c|}
\hline circRNA ID & Location & $\begin{array}{l}\text { Gene } \\
\text { symbol }\end{array}$ & Targets \\
\hline novel_circ_000125 & $\operatorname{chr} 1$ & Agap1 & $\begin{array}{l}\text { mmu-miR-15b-5p; mmu-miR-16-5p; mmu-miR-1907; mmu-miR-195a-5p; } \\
\text { mmu-miR-195b }\end{array}$ \\
\hline novel_circ_000176 & $\operatorname{chr} 1$ & Camsap2 & $\begin{array}{l}\text { mmu-let-7a-2-3p; mmu-miR-103-3p; mmu-miR-107-3p; mmu-miR-124-5p; } \\
\text { mmu-miR-139-5p }\end{array}$ \\
\hline novel_circ_000813 & chr 12 & Psma6 & $\begin{array}{l}\text { mmu-miR-1197-5p; mmu-miR-154-3p; mmu-miR-1903; mmu-miR-1912-3p; } \\
\text { mmu-miR-199a-3p }\end{array}$ \\
\hline novel_circ_002426 & $\operatorname{chr} 3$ & Fmo5 & mmu-let-7a-5p; mmu-let-7c-5p; mmu-let-7d-5p; mmu-let-7f-5p; mmu-let-7g-5p \\
\hline novel_circ_002561 & $\operatorname{chr} 4$ & Hemgn & $\begin{array}{l}\text { mmu-miR-103-3p; mmu-miR-105; mmu-miR-107-5p; mmu-miR-1197-5p; } \\
\text { mmu-miR-1198-5p }\end{array}$ \\
\hline novel_circ_002569 & $\operatorname{chr} 4$ & Abca1 & $\begin{array}{l}\text { mmu-let-7a-2-3p; mmu-miR-103-1-5p; mmu-miR-103-2-5p; mmu-miR-1193-5p; } \\
\text { mmu-miR-135a-5p }\end{array}$ \\
\hline novel_circ_002574 & $\operatorname{chr} 4$ & Tmem245 & $\begin{array}{l}\text { mmu-miR-1192; mmu-miR-1247-5p; mmu-miR-127-5p; mmu-miR-1306-5p; } \\
\text { mmu-miR-153-5p }\end{array}$ \\
\hline novel_circ_002674 & $\operatorname{chr} 4$ & Zmym4 & $\begin{array}{l}\text { mmu-miR-152-5p; mmu-miR-1938; mmu-miR-1955-3p; mmu-miR-1958; } \\
\text { mmu-miR-1964-5p }\end{array}$ \\
\hline novel_circ_002813 & $\operatorname{chr} 5$ & Ppp1cb & $\begin{array}{l}\text { mmu-miR-1264-3p;mmu-miR-1298-3p;mmu-miR-1306-5p;mmu-miR-130b-5p; } \\
\text { mmu-miR-137-5p }\end{array}$ \\
\hline novel_circ_003376 & $\operatorname{chr} 7$ & Pik $3 c 2 a$ & $\begin{array}{l}\text { mmu-let-7f-2-3p; mmu-miR-103-1-5p; mmu-miR-103-2-5p; mmu-miR-107-5p; } \\
\text { mmu-miR-1191a }\end{array}$ \\
\hline novel_circ_003491 & $\operatorname{chr} 8$ & Nsd3 & $\begin{array}{l}\text { mmu-miR-1224-3p; mmu-miR-137-5p; mmu-miR-188-5p; mmu-miR-194-1-3p; } \\
\text { mmu-miR-214-5p }\end{array}$ \\
\hline
\end{tabular}

novel_circ_000125, mm9_circ_017649; novel_circ_002813, mm9_circ_018676.

others), cell cycle and division (cyclin E1, anaphase promoting complex subunit 16, cyclin T2 and cyclin-dependent kinase 6 and others), stem cell (Nanog), metabolism (insulin-induced gene 2, hepatocyte nuclear factor 4 gamma, insulin-like growth factor 1 and ATP binding cassette subfamily D and others) and inflammation [interleukin (IL)11, IL6 signal transducer, IL5 receptor subunit alpha and tumor necrosis factor alpha-induced protein 3] were selected to establish the circRNA-miRNA-mRNA network. As presented in Fig. 8A, novel_circ_002561 was predicted to combine with 41 miRNAs to regulate the expression of 104 target genes. Some of the 41 miRNAs were mmu-miR-96-5p, mmu-miR-666-3p, mmu-miR-183-5p, mmu-miR-141-3p, mmu-miR-296-5p and mmu-miR-200a-3p. The networks of 2 further circRNAs, novel_circ_000176 and novel_circ_000189 are presented in Fig. 8B and C, respectively. The network of all the target genes of novel_circ_002561, novel_circ_000176 and novel_ circ_000189 are presented in Fig. S6. The networks of the $\overline{6}$ known differentially expressed circRNAs (novel_circ_000125, novel_circ_000596, novel_circ_000649, novel_circ_002754, novel_circ_002813 and novel_circ_003291) and 6 other randomly selected novel differentially expressed circRNAs are presented in Figs. S7-S8.

\section{Discussion}

EpCAM is a cell-cell adhesion molecule, which serves important roles in cell signaling, proliferation, differentiation, formation and maintenance of organ morphology (1). The phonotype of EpCAM knockout mice primarily manifests the symptoms of intestinal defects, with abnormalities in tight junctions and barrier functions of the intestinal epithelium (2). Recently, EpCAM was identified as a novel marker of cancer stem cells in HCC. Additionally, EpCAM-positive HCC cells exhibited hepatic cancer stem cell-like traits that could initiate highly invasive $\mathrm{HCC}$ in severe combined immunodeficient mice (33).

In the current study, the results of immunofluorescence staining demonstrated that EpCAM was mainly expressed on the liver cholangiocytes of WT mice at E18.5 and P0. However, this expression was completely eradicated in the livers of $\mathrm{EpCAM}^{-/}$mice, indicating that a successful knockout mouse model was generated in the present study. RT-qPCR analysis of genes associated with liver development revealed increased CD34 levels in the livers of $\mathrm{EpCAM}^{-/}$mice, compared with WT mice. CD34 is a marker of hepatic oval cells, resembling hepatic progenitor cells (HPCs), located in the periportal region of the liver (36). In response to liver injury, HPCs become involved in the proliferation and differentiation of liver cells (34). Since the expression of CD34 increased in the EpCAM knockout mice in the current study, this was confirmed to resemble a type of liver injury. In addition, PDCs have long been considered a type of putative liver stem/progenitor cell, and EpCAM-positive PDCs with genetic alterations induced by chemicals have been determined to develop into HCC, resembling human cholangiolocellular 
Table II. Basic information and predicted targets of down-regulated circRNAs.

\begin{tabular}{|c|c|c|c|}
\hline circRNA ID & Location & $\begin{array}{l}\text { Gene } \\
\text { symbol }\end{array}$ & Targets \\
\hline novel_circ_000085 & chr 1 & Kans111 & $\begin{array}{l}\text { mmu-let-7a-2-3p; mmu-let-7c-1-3p; mmu-let-7j; mmu-miR-101a-3p; } \\
\text { mmu-miR-101c }\end{array}$ \\
\hline novel_circ_000161 & $\operatorname{chr} 1$ & Tmcc2 & $\begin{array}{l}\text { mmu-miR-1188-5p; mmu-miR-1190; mmu-miR-1258-5p; mmu-miR-129-1-3p; } \\
\text { mmu-miR-129-2-3p }\end{array}$ \\
\hline novel_circ_000189 & $\operatorname{chr} 1$ & Rnf2 & $\begin{array}{l}\text { mmu-miR-1933-5p; mmu-miR-19b-1-5p; mmu-miR-19b-2-5p; mmu-miR-302b-5p; } \\
\text { mmu-miR-302c-5p }\end{array}$ \\
\hline novel_circ_000596 & $\operatorname{chr} 11$ & Smg6 & $\begin{array}{l}\text { mmu-miR-105; mmu-miR-107-5p; mmu-miR-1190; mmu-miR-1224-5p; } \\
\text { mmu-miR-1231-5p }\end{array}$ \\
\hline novel_circ_000649 & $\operatorname{chr} 11$ & Vezf1 & $\begin{array}{l}\text { mmu-miR-106a-3p; mmu-miR-1187; mmu-miR-1197-3p; mmu-miR-132-5p; } \\
\text { mmu-miR-134-5p }\end{array}$ \\
\hline novel_circ_001018 & chr 13 & Klhl3 & $\begin{array}{l}\text { mmu-miR-1251-3p; mmu-miR-133a-3p; mmu-miR-133b-3p; mmu-miR-133c; } \\
\text { mmu-miR-135a-5p }\end{array}$ \\
\hline novel_circ_002754 & $\operatorname{chr} 4$ & Rere & $\begin{array}{l}\text { mmu-miR-181d-3p; mmu-miR-1964-5p; mmu-miR-28a-5p; mmu-miR-666-3p; } \\
\text { mmu-miR-6929-3p }\end{array}$ \\
\hline novel_circ_002908 & $\operatorname{chr} 5$ & Aff1 & $\begin{array}{l}\text { mmu-miR-1197-3p; mmu-miR-1198-5p; mmu-miR-137-3p; mmu-miR-145a-3p; } \\
\text { mmu-miR-145a-5p }\end{array}$ \\
\hline novel_circ_003147 & $\operatorname{chr} 6$ & Slc41a3 & $\begin{array}{l}\text { mmu-miR-1197-5p; mmu-miR-1943-5p; mmu-miR-29a-3p; mmu-miR-29b-3p; } \\
\text { mmu-miR-29c-3p }\end{array}$ \\
\hline novel_circ_003291 & $\operatorname{chr} 7$ & Gas2 & $\begin{array}{l}\text { mmu-miR-124-3p; mmu-miR-1904; mmu-miR-207; mmu-miR-210-5p; } \\
\text { mmu-miR-24-3p }\end{array}$ \\
\hline novel_circ_003375 & $\operatorname{chr} 7$ & $\operatorname{Pik} 3 \mathrm{c} 2 \mathrm{a}$ & $\begin{array}{l}\text { mmu-miR-1898; mmu-miR-1904; mmu-miR-216b-5p; mmu-miR-22-3p; } \\
\text { mmu-miR-300-5p }\end{array}$ \\
\hline novel_circ_003479 & $\operatorname{chr} 8$ & Efnb2 & $\begin{array}{l}\text { mmu-let-7a-1-3p; mmu-let-7c-2-3p; mmu-miR-105; mmu-miR-1192; } \\
\text { mmu-miR-1224-3p }\end{array}$ \\
\hline
\end{tabular}

novel_circ_000596,mm9_circ_017983; novel_circ_000649,mm9_circ_015947; novel_circ_002754,mm9_circ_013483; novel_circ_003291, mm9_circ_003692.

carcinomas (CLCs). During this process, the Wnt signaling pathway was demonstrated to be specifically upregulated in the CLC components of PDC-derived HCC (10). The results of the present study revealed that the expression of Axin2, an important component and target gene of the Wnt signaling pathway, was significantly decreased in the livers of EpCAM ${ }^{-1-}$ mice. This result indicated that EpCAM may interact with the Wnt signaling pathway to play an important role in the liver development and liver diseases. Furthermore, the present results revealed the distinct expression of circRNAs in the livers of EpCAM knockout mice, such as novel_circ_002561, which could regulate the transcription of Wnt signaling related genes (Wnt3a, Lgr5, Tcf3 and Tcf4, and others). It was therefore hypothesized that EpCAM may regulate liver development via certain circRNAs.

circRNAs act as 'sponges' for microRNAs and RNA binding proteins to regulate target gene expression $(16,35,36)$. circRNAs have recently gained interest due to their complex involvement in the regulation of transcriptional processes their important roles in human diseases and their potential to serve as biomarkers and potential clinical targets (37). Although circRNAs modulate transcription and interfere with splicing, the expression pattern and functions of the majority of circRNAs remain largely unexplored. Therefore, the study of circRNAs and their dynamic expression patterns and complicated regulatory networks in different biological processes and diseases will help to further classify them (38). In the present study, high-throughput sequencing identified 3,984 circRNAs, including 292 known circRNAs and 3,692 novel circRNAs in the liver of WT and $\mathrm{EpCAM}^{-/-}$mice. The current study also identified 11 upregulated and 12 downregulated circRNAs in the livers of $\mathrm{EpCAM}^{-/-}$mice compared with the WT group. Within these circRNAs, 6 were known circRNAs and 17 were novel circRNAs. The results of GO analysis revealed that the majority of source genes of the differentially expressed circRNAs were associated with cellular process, single-organism process, metabolic process and developmental process. These data were consistent with the identification of development associated genes via RT-qPCR. These results demonstrated the important role of EpCAM in liver development.

$\mathrm{H} \& \mathrm{E}$ staining revealed reduced cytoplasmic vacuolation of the liver, in $\mathrm{EpCAM}^{-/-}$mice, suggesting defects in hepatic glycogen storage (28). The analysis of glycogen-associated genes then identified two UDP-glucose pyrophosphorylase genes (UGP1 and UGP2), which are essential for sucrose and polysaccharide synthesis (39). The results of the current study also revealed that the expression of UGP2 was decreased 

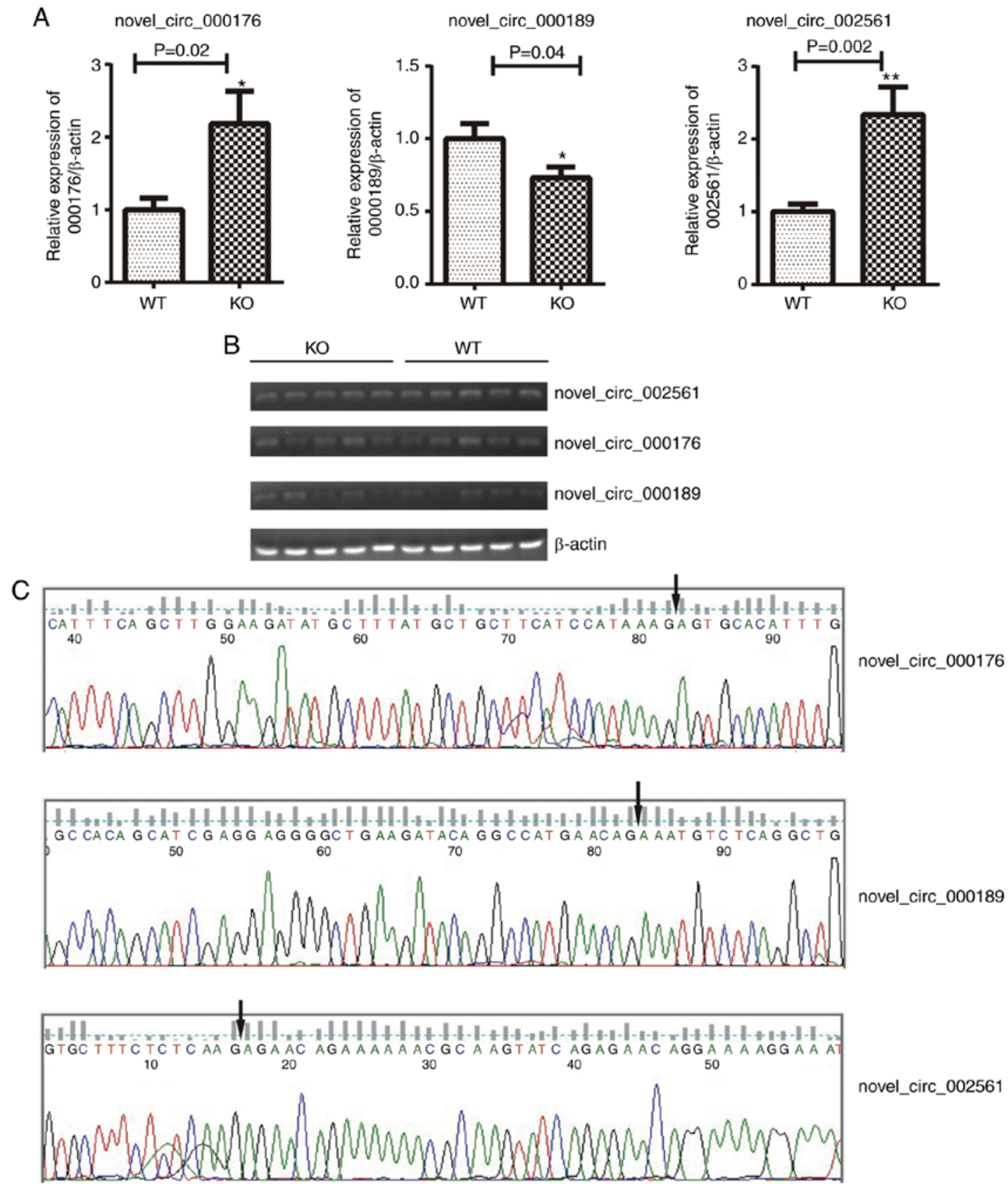

novel_circ_002561

Figure 6. Validation of the circRNA expression profiles of the livers of EpCAM ${ }^{-/}$mice. (A) The expression of novel_circ_002561, novel_circ_000176 and novel_circ_000189 was determined. (B) RT-q PCR products were visualized via electrophoresis. (C) RT-qPCR products were confirmed via Sanger sequencing. ${ }^{\mathrm{P}}<0.05$ and ${ }^{* *} \mathrm{P}<0.01$, vs. WT mice. circRNA, circular RNA; RT-qPCR, reverse transcription-quantitative PCR; EpCAM, epithelial cell adhesion molecule; WT, wild-type.

in the livers of $\mathrm{EpCAM}^{-/-}$mice. In addition, the expression of SLC2A2 was increased in the $\mathrm{EpCAM}^{-/}$livers. SLC2A2 encodes the glucose transporter 2 (GLUT2), and a defect may lead to neonatal diabetes, hepatomegaly and renal Fanconi syndrome (40). The results of the current study demonstrated that EpCAM affected glycogen synthesis and genes associated with diseases of glycogen storage. However, the underlying mechanisms warrant further investigation in future studies. The results of KEGG pathway analysis demonstrated that the top 20 pathways of differentially expressed circRNA source genes included mRNA surveillance, fat digestion and absorption and insulin signaling pathways. These bioinformatics data were consistent with the results of RT-qPCR and glycogen
H\&E staining, indicating that EpCAM may also be involved in liver glycogen metabolism.

The results of H\&E staining also revealed that the livers of EpCAM $^{-/}$mice at $\mathrm{P} 4$ contained marked vacuolation compared with the livers of WT mice. EpCAM ${ }^{-/}$mice also manifested intestinal barrier defects and died shortly after birth as a result of intestinal erosion, showing similar phenotypes with a previous study (2). It is hypothesized that $\mathrm{EpCAM}^{-/-}$mice exhibiting intestinal defects may have difficulties in digesting and absorbing food and energy, which affected liver glycogen metabolism, causing vacuolation. This hypothesis was supported by the data of the present study. However, the underlying mechanisms require further investigation. 
A

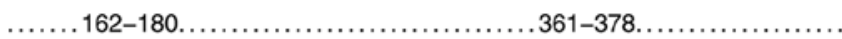

......----UGUUUGGAUAUGUUAAGA........AUGAAGCGCAUCAGGAAA. .............novel_circ_000189

.... UCUUUCGUAAGG--GUACAAUUUCA. . . . . . . . . . . . . . . . mmu-miR-302b-5p

$\ldots \ldots \ldots \ldots \ldots \ldots \ldots \ldots \ldots$ AAAAGACGGAGAGAAGUCCUUA. . . . . . mmu-miR-691

B

... 76-95. . . . . . . . . . . . . . . . . . . . . . . .

.... GCAUG--UGCUCAACAGUAUUC. ...... --AGAUAUGCUUUAUGCUGCUU. . . . . . . . . . novel_circ_000176

$\ldots$. AGUAGUAAUGGUCCGUCAUAAU. . . . . . . . . . . . . . . . . . . . . mmu-miR-200b-3p

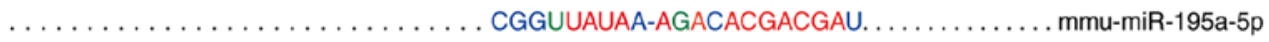

C



Figure 7. Examples of miRNA target sites of novel_circ_000189, novel_circ_000176 and novel_circ_002561. (A) The mmu-miR-302b-5p and mmu-miR-691 binding sites of novel_circ_000189. (B) The mmu-miR-200b-3p and mmu-miR-195a-5p binding sites of novel_circ_000176. (C) The mmu-miR-200a-3p and mmu-miR-466b-5p binding sites of novel_circ_002561. Red represents the complementary sequence of circRNA with miRNAs, green represents the binding of Guanine and Uracil (GU), and blue demonstrates the section of miRNA that cannot bind to circRNA. circRNA direction: 5'-3', miRNA direction 3'-5'. miRNA or miR, microRNA; circRNA, circular RNA.

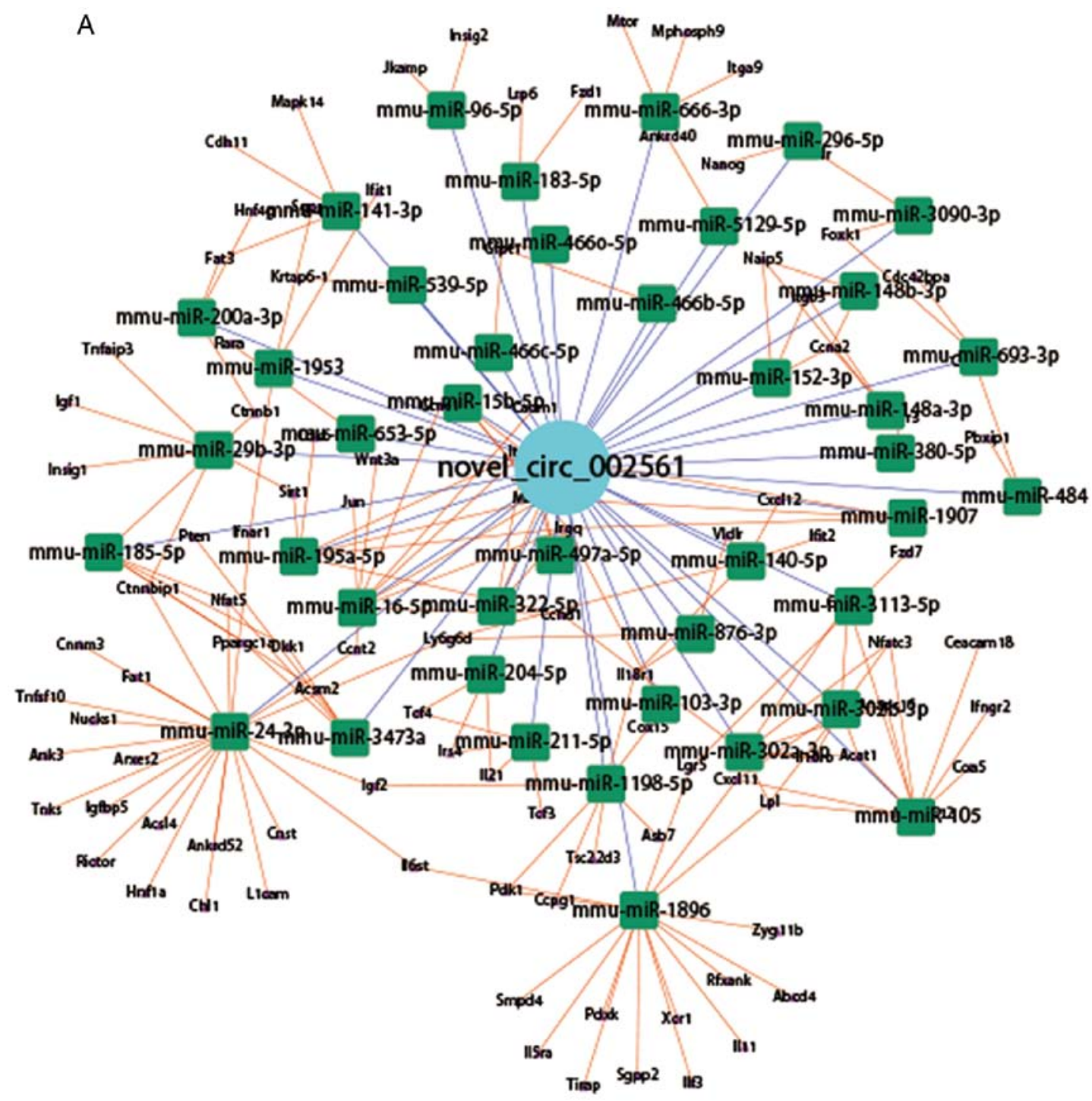

Figure 8. Prediction of the target associations of the circRNA-microRNA-mRNA network. circRNA-microRNA-mRNA networks of the target genes associated with Wnt signaling pathway, cell junctions, cell cycle and division, stem cell, metabolism and inflammation of (A) novel_circ_002561. 

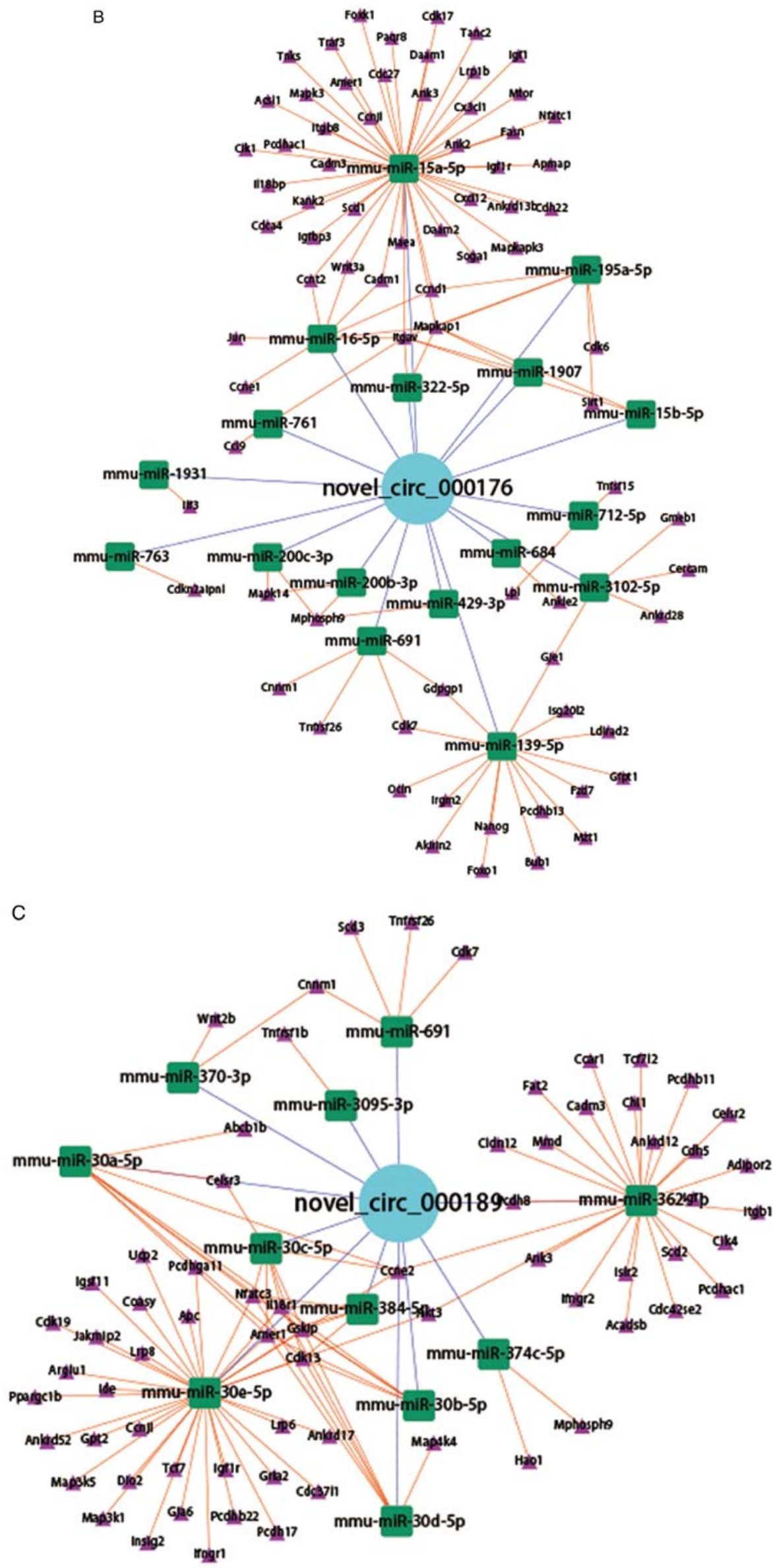

Figure 8. Continued. Prediction of the target associations of the circRNA-microRNA-mRNA network. circRNA-microRNA-mRNA networks of the target genes associated with Wnt signaling pathway, cell junctions, cell cycle and division, stem cell, metabolism and inflammation of (B) novel_circ_000176 and (C) novel_circ_000189. circRNA, circular RNA. 
To further study the functions and mechanisms of liver EpCAM, the target associations of the circRNA-miRNAmRNA networks was predicted. Based on experimental results, target genes associated with the Wnt signaling pathway, cell junctions, cell cycle and division, stem cells, metabolism and inflammation were selected to establish the circRNA-miRNA-mRNA network. The results identified Novel_circ_002561, located in chromosome 4, whose expression was up-regulated in the livers of $\mathrm{EpCAM}^{-1-}$ mice. The circRNA-microRNA-mRNA network of novel_circ_002561, its target genes and target microRNAs were also established. From network analysis, the regulatory association was clearly demonstrated, providing directions for further analysis.

In circRNAs profiling, 6 known circRNAs were differentially expressed. Novel_circ_000125 (mm9_ circ_017649) and novel_circ_002813 (mm9_circ_018676) were up-regulated in the livers of $\mathrm{EpCAM}^{-/-}$mice, while novel_circ_000596 (mm9_circ_017983), novel_circ_000649 (mm9_circ_015947), novel_circ_002754 (mm9_ circ_013483) and novel_circ_003291 (mm9_circ_003692) were down-regulated. As determined by Memczak et al (27), novel_circ_000125 and novel_circ_003291 were detected in the brains and heads of mice, novel_circ_000596 was detected in the embryonic stem cells (ES) and heads of mice, novel_circ_000649 was detected in the heads of mice, novel_circ_002754 was detected in the ES and brains of mice, and novel_circ_0002813 was detected in the ES, brains and heads of mice. The current study determined that these circRNAs were also expressed in the livers of WT mice at the P0 stage and that their expression was altered in the livers of $\mathrm{EpCAM}^{-/}$mice. However, their functions, mechanisms and homologous human sequences remain obscure. The circRNAs determined in the present study may provide useful information for further study.

In conclusion, the current study revealed that the expression of certain development and glycogen-associated genes was altered in the livers of $\mathrm{EpCAM}^{--}$mice. The livers of $\mathrm{EpCAM}^{-/-}$ mice exhibited glycogen shortages at E18.5 and vacuolation at P4. Based on RNA sequencing, a circRNA expression profile of $\mathrm{EpCAM}^{-/-}$murine livers was established. Several novel circRNAs were identified, and the circRNA-miRNA-mRNA regulatory network was established. These results may provide important information and direction for the future development of novel targets for the treatment of liver disease.

\section{Acknowledgements}

The authors would like to express their gratitude to Professor Yaacov Ben-David (University of Toronto) for markedly revising the text of the manuscript.

\section{Funding}

This study was supported by the National Natural Science Foundation of China (grant nos. 31671520 and 81803912), the Science and Technology Project of Guangdong Province (grant nos. 2016B050501003 and 2017B050504005), the Scientific Research Project of the Administration of Traditional Chinese Medicine of Guangdong Province (grant no. 20182079), the Characteristic Innovation Project (Natural Science) of the Education Department of Guangdong Province and the 'Innovation Strong School Project' of Guangdong Pharmaceutical University (grant no. 2017KTSCX102), and the Science and Technology Project of Yue-Xiu District of Guangzhou (grant no. 2018-WS-011).

\section{Availability of data and materials}

The datasets generated and analyzed during the current study are not publicly available due to the reason that part of the dataset has not been analyzed and published yet, but are available from the corresponding author on reasonable request.

\section{Authors' contributions}

ZL, LL and JG designed the study and conceived the report. YY analyzed and interpreted the results of RNA sequencing, wrote the first draft of the manuscript and revised it critically. SL, GC and LH established the mouse model. FY, YLi, LY, WL and YLe performed the molecular experiments, including RT-qPCR and H\&E staining. YLe also created the figures. All authors read and approved the final manuscript.

\section{Ethics approval and consent to participate}

All animal experiments were approved by the Committee on the Laboratory Animal Care and Use of Guangdong Pharmaceutical University.

\section{Patient consent for publication}

Not applicable.

\section{Competing interests}

The authors declare that they have no competing interests.

\section{References}

1. Huang L, Yang Y, Yang F, Liu S, Zhu Z, Lei Z and Guo J: Functions of EpCAM in physiological processes and diseases (Review). Int J Mol Med 42: 1771-1785, 2018.

2. Lei Z, Maeda T, Tamura A, Nakamura T, Yamazaki Y, Shiratori H, Yashiro K, Tsukita S and Hamada H: EpCAM contributes to formation of functional tight junction in the intestinal epithelium by recruiting claudin proteins. Dev Biol 371: 136-145, 2012.

3. Guerra E, Lattanzio R, La Sorda R, Dini F, Tiboni GM, Piantelli $M$ and Alberti S: mTrop1/Epcam knockout mice develop congenital tufting enteropathy through dysregulation of intestinal E-cadherin/ $\beta$-catenin. PLoS One 7: e49302, 2012.

4. Mueller JL, McGeough MD, Peña CA and Sivagnanam M: Functional consequences of EpCam mutation in mice and men. Am J Physiol Gastrointest Liver Physiol 306: G278-G288, 2014.

5. Yousaf M, Tayyeb A and Ali G: Expression profiling of adhesion proteins during prenatal and postnatal liver development in rats. Stem Cells Cloning 10: 21-28, 2017.

6. Tanaka M, Okabe M, Suzuki K, Kamiya Y, Tsukahara Y, Saito S and Miyajima A: Mouse hepatoblasts at distinct developmental stages are characterized by expression of EpCAM and DLK1: Drastic change of EpCAM expression during liver development. Mech Dev 126: 665-676, 2009.

7. Okabe M, Tsukahara Y, Tanaka M, Suzuki K, Saito S, Kamiya Y, Tsujimura T, Nakamura K and Miyajima A: Potential hepatic stem cells reside in EpCAM+ cells of normal and injured mouse liver. Development 136: 1951-1960, 2009. 
8. Schmelzer E, Zhang L, Bruce A, Wauthier E, Ludlow J, Yao HL, Moss N, Melhem A, McClelland R, Turner W, et al: Human hepatic stem cells from fetal and postnatal donors. J Exp Med 204: 1973-1987, 2007.

9. Lu H, Ma J, Yang Y, Shi W and Luo L: EpCAM is an endoderm-specific Wnt derepressor that licenses hepatic development Dev Cell 24: 543-553, 2013.

10. Matsumoto T, Takai A, Eso Y, Kinoshita K, Manabe T, Seno H, Chiba T and Marusawa H: Proliferating EpCAM-positive ducta cells in the inflamed liver give rise to hepatocellular carcinoma. Cancer Res 77: 6131-6143, 2017.

11. Yamashita T, Forgues M, Wang W, Kim JW, Ye Q, Jia H, Budhu A, Zanetti KA, Chen Y, Qin LX, et al: EpCAM and alpha-fetoprotein expression defines novel prognostic subtypes of hepatocellular carcinoma. Cancer Res 68: 1451-1461, 2008.

12. Yamashita T, Ji J, Budhu A, Forgues M, Yang W, Wang HY, Jia H, Ye Q, Qin LX, Wauthier E, et al: EpCAM-positive hepatocellular carcinoma cells are tumor-initiating cells with stem/progenitor cell features. Gastroenterology 136: 1012-1024, 2009.

13. Ji J, Yamashita T, Budhu A, Forgues M, Jia HL, Li C, Deng C, Wauthier E, Reid LM, Ye QH, et al: Identification of microRNA-181 by genome-wide screening as a critical player in EpCAM-positive hepatic cancer stem cells. Hepatology 50: 472-480, 2009

14. Mani SK, Zhang H, Diab A, Pascuzzi PE, Lefrançois L, Fares N, Bancel B, Merle P and Andrisani O: EpCAM-regulated intramembrane proteolysis induces a cancer stem cell-like gene signature in hepatitis B virus-infected hepatocytes. J Hepatol 65 : 888-898, 2016.

15. Song Y, Liu C, Liu X, Trottier J, Beaudoin M, Zhang L, Pope C, Peng G, Barbier O, Zhong X, et al: $\mathrm{H} 19$ promotes cholestatic liver fibrosis by preventing ZEB1-mediated inhibition of epithelial cel adhesion molecule. Hepatology 66: 1183-1196, 2017.

16. Lu C, Sun X, Li N, Wang W, Kuang D, Tong P, Han Y and Dai J: CircRNAs in the tree shrew (Tupaia belangeri) brain during postnatal development and aging. Aging (Albany NY) 10: 833-852, 2018

17. Maiese K: Disease onset and aging in the world of circular RNAs. J Transl Sci 2: 327-329, 2016

18. Luo Q, Zhang L, Li X, Fu B, Deng Z, Qing C, Su R, Xu J, Guo Y, Huang Z and Li J: Identification of circular RNAs hsa_circ_0044235 in peripheral blood as novel biomarkers for rheumatoid arthritis. Clin Exp Immunol 194: 118-124, 2018.

19. Wang $X$ and Fang L: Advances in circular RNAs and their roles in breast cancer. J Exp Clin Cancer Re 37: 206, 2018.

20. Holdt LM, Kohlmaier A and Teupser D: Molecular functions and specific roles of circRNAs in the cardiovascular system. Noncoding RNA Res 3: 75-98, 2018.

21. Yu CX and Sun S: An emerging role for circular RNAs in osteoarthritis. Yonsei Med J 59: 349-355, 2018

22. Yao R, Zou H and Liao W: Prospect of circular RNA in hepatocellular carcinoma: A novel potential biomarker and therapeutic target. Front Oncol 8: 332, 2018.

23. Guo XY, He CX, Wang YQ, Sun C, Li GM, Su Q, Pan Q and Fan JG: Circular RNA profiling and bioinformatic modeling identify its regulatory role in hepatic steatosis. Biomed Res Int 2017: 5936171, 2017

24. Guo J, Zhou Y, Cheng Y, Fang W, Hu G, Wei J, Lin Y, Man Y, Guo L, Sun M, et al: Metformin-induced changes of the coding transcriptome and non-coding RNAs in the livers of non-alcoholic fatty liver disease mice. Cell Physiol Biochem 45: 1487-1505, 2018.
25. Langmead B and Salzberg SL: Fast gapped-read alignment with Bowtie 2. Nat Methods 9: 357-359, 2012.

26. Kim D, Pertea G, Trapnell C, Pimentel H, Kelley R and Salzberg SL: TopHat2: Accurate alignment of transcriptomes in the presence of insertions, deletions and gene fusions. Genome Biol 14: R36, 2013.

27. Memczak S, Jens M, Elefsinioti A, Torti F, Krueger J, Rybak A, Maier L, Mackowiak SD, Gregersen LH, Munschauer M, et al: Circular RNAs are a large class of animal RNAs with regulatory potency. Nature 495: 333-338, 2013.

28. Choi E, Zhang X, Xing $\mathrm{C}$ and $\mathrm{Yu} \mathrm{H}$ : Mitotic checkpoint regulators control insulin signaling and metabolic homeostasis. Cell 166: 567-581, 2016.

29. Stygar D, Andrare D, Bażanów B, Chełmecka E, Sawczyn T, Skrzep-Poloczek B, Olszańska E, Karcz KW and Jochem J: The impact of DJOS surgery, a high fat diet and a control diet on the enzymes of glucose metabolism in the liver and muscles of Sprague-Dawley rats. Front Physiol 10: 571, 2019.

30. Adeva-Andany MM, González-Lucán M, Donapetry-García C, Fernández-Fernández $\mathrm{C}$ and Ameneiros-Rodríguez E: Glycogen metabolism in humans. BBA Clin 5: 85-100, 2016.

31. Zhang DD, Zhang JG, Wang YZ, Liu Y, Liu GL and Li XY: Per-Arnt-Sim Kinase (PASK): An emerging regulator of mammalian glucose and lipid metabolism. Nutrients 7: 7437-7450, 2015.

32. Hajiaghaalipour F, Khalilpourfarshbafi M and Arya A Modulation of glucose transporter protein by dietary flavonoids in type 2 diabetes mellitus. Int J Biol Sci 11: 508-524, 2015.

33. Terris B, Cavard C and Perret C: EpCAM, a new marker for cancer stem cells in hepatocellular carcinoma. J Hepatol 52: 280-281, 2010.

34. Weiss TS and Dayoub R: Thy-1 (CD90)-positive hepatic progenitor cells, hepatoctyes, and non-parenchymal liver cells isolated from human livers. Methods Mol Biol 1506: 75-89, 2017.

35. Bose R and Ain R: Regulation of transcription by circular RNAs. Adv Exp Med Biol 1087: 81-94, 2018.

36. Hansen TB, Jensen TI, Clausen BH, Bramsen JB, Finsen B, Damgaard CK and Kjems J: Natural RNA circles function as efficient microRNA sponges. Nature 495: 384-388, 2013.

37. Franz A, Rabien A, Stephan C, Ralla B, Fuchs S, Jung K and Fendler A: Circular RNAs: A new class of biomarkers as a rising interest in laboratory medicine. Clin Chem Lab Med 56: 1992-2003, 2018.

38. Li X, Yang L and Chen LL: The biogenesis, functions, and challenges of circular RNAs. Mol Cell 71: 428-442, 2018.

39. Wang Q, Yang ZL, Zou Q, Yuan Y, Li J, Liang L, Zeng G and Chen S: SHP2 and UGP2 are biomarkers for progression and poor prognosis of gallbladder cancer. Cancer Invest 34: 255-264, 2016.

40. Khandelwal P, Sinha A, Jain V, Houghton J, Hari P and Bagga A: Fanconi syndrome and neonatal diabetes: Phenotypic heterogeneity in patients with GLUT2 defects. CEN Case Rep 7: 1-4, 2018.

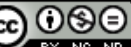

This work is licensed under a Creative Commons Attribution-NonCommercial-NoDerivatives 4.0 International (CC BY-NC-ND 4.0) License. 